\title{
Ist die Herzfrequenz bei Herzinsuffizienz-Patienten in Deutschland ausreichend kontrolliert?
}

\author{
Ergebnisse einer nationalen Beobachtungsstudie (INDICATE)
}

C. Zugck ${ }^{1}$, P. Martinka², G. Stöckl², C. Tschöpe ${ }^{3}$, S. Störk ${ }^{4}$

Institute

1 Internistische Gemeinschaftspraxis Steiner Thor, Straubing

2 Servier Deutschland $\mathrm{GmbH}$, München

3 Medizinische Klinik für Kardiologie und Pulmologie, Campus Benjamin Franklin, Charité Universitätsmedizin, Berlin

4 Deutsches Zentrum für Herzinsuffizienz, Universität Würzburg
Korrespondenz

Prof. Dr. Christian Zugck

Internistische Gemeinschaftspraxis Steiner Thor,

Bahnhofstraße 1

94315 Straubing

zugck@internisten-steinerthor.de

\section{Zusammenfassung}

Einführung | Eine erhöhte Ruheherzfrequenz geht mit einer gesteigerten Morbidität und Mortalität bei Patienten mit chronischer systolischer Herzinsuffizienz (CHI) einher. Eine Reduktion der Herzfrequenz verbessert die kardiovaskuläre Prognose dieser Patienten. Die Senkung der Herzfrequenz besitzt deshalb einen hohen Stellenwert in der Therapie der $\mathrm{CHI}$ und ist dementsprechend auch in den aktuellen europäischen Leitlinien zur Herzinsuffizienz abgebildet.

Methoden I Um die aktuelle Versorgungssituation von ambulanten Patienten mit CHI (dokumentierte linksventrikuläre systolische Dysfunktion) in Deutschland zu analysieren, wurde die multizentrische bundesweite Querschnitterhebung INDICATE (ScreenINg of toDay's patlents with Chronic systolic heArT failurE) durchgeführt. Hierzu sollten von Februar bis Juni 2012 durch 793 kardiologische Facharztpraxen jeweils 20 konsekutive Patienten mit diagnostizierter $\mathrm{CHI}$ in die Untersuchung eingeschlossen und ausführ-

\section{Einleitung}

Inzidenz und Prävalenz der Herzinsuffizienz haben in den vergangenen Jahrzehnten in Deutschland wie auch in anderen westlichen Industrieländern stetig zugenommen. Man nimmt an, dass derzeit ca. 1,8 Millionen Menschen in Deutschland an einer chronischen Herzinsuffizienz mit linksventrikulärer Dysfunktion leiden [3] und jährlich etwa 300000 Patienten neu hinzukommen [10].

Eine Herzinsuffizienz ist mit einer progredienten Abnahme von Lebensqualität und körperlicher Leistungsfähigkeit verbunden. Häufige Hospitalisierungen, lange Phasen von Arbeitsunfähigkeit sowie eine hohe Rate an krankheitsbedingter, längerfristiger Erwerbsunfähigkeit haben erhebliche gesundheitsökonomische und gesamtwirtschaftliche Konsequenzen.

Kürzlich berichtete die europaweite ESC-HF PilotStudie über die Einjahres-Prognose eines großen Kollektivs von Patienten mit chronischer stabiler Herzinsuffizienz $(n=3226)$. Sie fand eine Mortali- lich mittels eines standardisierten Fragebogens dokumentiert werden.

Ergebnisse I Die Herzinsuffizienz war bei $88 \%$ der 15148 charakterisierten Patienten seit mehr als 6 Monaten bekannt. Die durchschnittliche Herzfrequenz im Kollektiv betrug $73 \pm 13 \mathrm{~min}^{-1}$. Dabei wiesen $42 \%$ der Patienten eine Herzfrequenz $\geq 75 \mathrm{~min}^{-1}$ auf. Eine Therapie mit Betarezeptorblockern erhielten $86 \%$ der Patienten, wobei eine höhere Betablockerdosis jedoch nicht mit niedrigerer Ruheherzfrequenz assoziiert war. Bei $27 \%$ der Patienten lag die Herzfrequenz bei $\geq 75 \mathrm{~min}^{-1}$, obwohl sie mindestens $50 \%$ der Betablocker-Zieldosis erhielten.

Folgerungen I INDICATE zeigt, dass in Deutschland ein erheblicher Anteil der ambulanten Patienten mit CHI trotz Betablocker-Therapie - unabhängig von der Dosierung - eine erhöhte Ruheherzfrequenz aufweist. Dies unterstreicht das Potenzial der Leitlinien-gestützten pharmakologischen Optimierung der Ruheherzfrequenz bei diesen Patienten. tät von $7,2 \%$ und eine Hospitalisierungsrate von 31,9\% [8]. Zu vergleichbaren Ergebnissen für diese Kennzahlen, nämlich 5,9\% und 22,7\%, kommt auch ein aktuelles italienisches Register $(n=3755)$ [17].

Eine erhöhte Ruheherzfrequenz ist ein wichtiger und therapeutisch gut beeinflussbarer Faktor, der die Morbidität und Mortalität von Patienten mit chronischer Herzinsuffizienz steigert [12, 13]. Zur Kontrolle der Herzfrequenz werden primär Betarezeptorblocker eingesetzt. Sie

- reduzieren die Belastung des linksventrikulären Myokards,

- dämpfen den adrenergen Antrieb,

- verbessern das myokardiale Remodelling und

- bedingen dadurch eine reduzierte kardiovaskuläre Morbidität und Mortalität [4].

Deshalb sind Betarezeptorblocker seit etlichen Jahren in internationalen Leitlinien als Prognoseverbessernde Standardtherapie bei Herzinsuffizienz empfohlen [9].

In der SHIFT-Studie (Systolic Heart Failure treatment with the If inhibitor Ivabradine Trial) redu- 
zierte die selektive Frequenzsenkung mit dem IfKanalblocker Ivabradin im Vergleich zu Placebo die kardiovaskuläre Ereignisrate von Patienten mit chronischer systolischer Herzinsuffizienz (18\% relative Risikoreduktion beim kombinierten Endpunkt) [15]. In SHIFT erhielten die Patienten randomisiert Ivabradin $(n=3241)$ oder Placebo $(n=3264)$ zusätzlich zu einer optimierten leitliniengerechten Basistherapie. Diese schloss auch Betablocker ein. Damit bestätigte SHIFT, dass bei Patienten mit chronisch systolischer Herzinsuffizienz eine erhöhte Herzfrequenz nicht nur ein Marker, sondern ein Risikofaktor für kardiovaskuläre Ereignisse ist, dessen Reduktion die Prognose dieser Patienten verbessert. Zwischenzeitlich wurde Ivabradin auch in die aktuellen europäischen Herzinsuffizienz-Leitlinien aus dem Jahr 2012 zur Behandlung symptomatischer Patienten mit
- erhöhter Herzfrequenz $\left(\geq 70 \mathrm{~min}^{-1}\right)$,

- höhergradig eingeschränkter linksventrikulärer Funktion (LVEF $\leq 35 \%)$ und

- optimierter Begleittherapie (inkl. ACE-

Hemmer, Betablocker und Aldosteron-Antagonisten)

aufgenommen [9].

Es stellt sich jedoch immer die grundlegende Frage, inwieweit sich die Daten aus randomisierten Studien mit entsprechend selektierten Patientenpopulationen in der Versorgungsrealität abbilden lassen. Nach Aufnahme der Betablocker in die Leitlinien zur Behandlung der Herzinsuffizienz Anfang des letzten Jahrzehnts wurde deshalb begonnen, komplementär zu randomisierten Studien auch Registerdaten zu erheben und in die klinische Bewertung mit einfließen zu lassen. Dabei zeigte sich eine zum großen Teil unzurei-

Tab. 1 Ausgewählte Patientencharakteristika inklusive wichtiger Begleiterkrankungen und kardiovaskulärer Medikation des Studienkollektivs. Werte angegeben als Mittelwert \pm Standardabweichung oder Prozent des Gesamt- bzw. des jeweiligen Subkollektivs.

ICD = Implantable Cardioverter Defibrillator;

CRT = Cardiac Resynchronization Therapy.

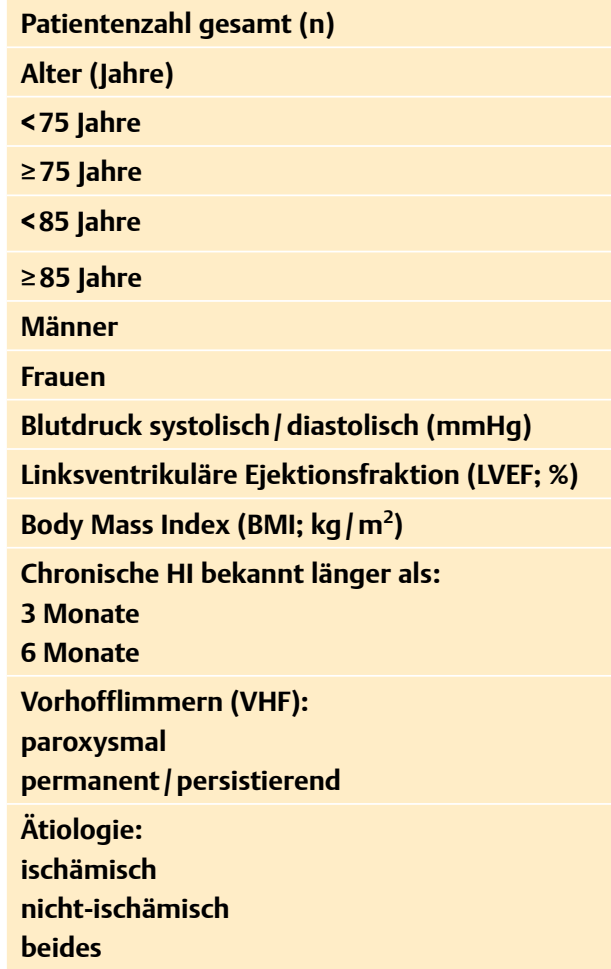

Zeichen von Dekompensation (periphere Ödeme, verschlechterte Dyspnoe, Aszites usw.) vorhanden

nicht vorhanden

keine Angabe

Koronare Herzkrankheit (KHK)

Myokardinfarkt in der Vorgeschichte

Herzschrittmacher (HS):

ICD

CRT

ICD/CRT

keine Typangabe

15148
$70 \pm 11$
$60 \%$
$40 \%$
$92 \%$
$8 \%$
$62 \%$
$38 \%$
$133 \pm 19 / 79 \pm 11$
$43 \pm 12$
$29 \pm 5$
$12 \%$
$88 \%$
$27 \%$
$26 \%$ (der Subgruppe VHF)
$74 \%$ (der Subgruppe VHF)

Begleiterkrankungen:
Arterielle Hypertonie
Hyperlipidämien
Diabetes mellitus
Chronische Nierenerkrankung
Chronisch obstruktive Lungenerkrankung (COPD)
Depression
Schilddrüsenerkrankungen
Periphere arterielle Verschlusskrankheit (pAVK)
Asthma bronchiale
Maligne Erkrankungen / Tumore
Anämie
Lebererkrankung
Demenz
Dialyse-Abhängigkeit

Kardiovaskuläre Medikation:

ACE-Hemmer

AT1-Rezeptorantagonisten

Betarezeptorenblocker

Mineralocorticoid-Rezeptorantagonisten

$23 \%$

Schleifendiuretika

$49 \%$

Thiaziddiuretika

$28 \%$

Herzglykoside

Calciumantagonisten

$21 \%$

Acetylsalicylsäure

$54 \%$

Langwirksame Nitrate

$9 \%$

(orale) Antikoagulantien

$28 \%$

Statine

Antidiabetika 

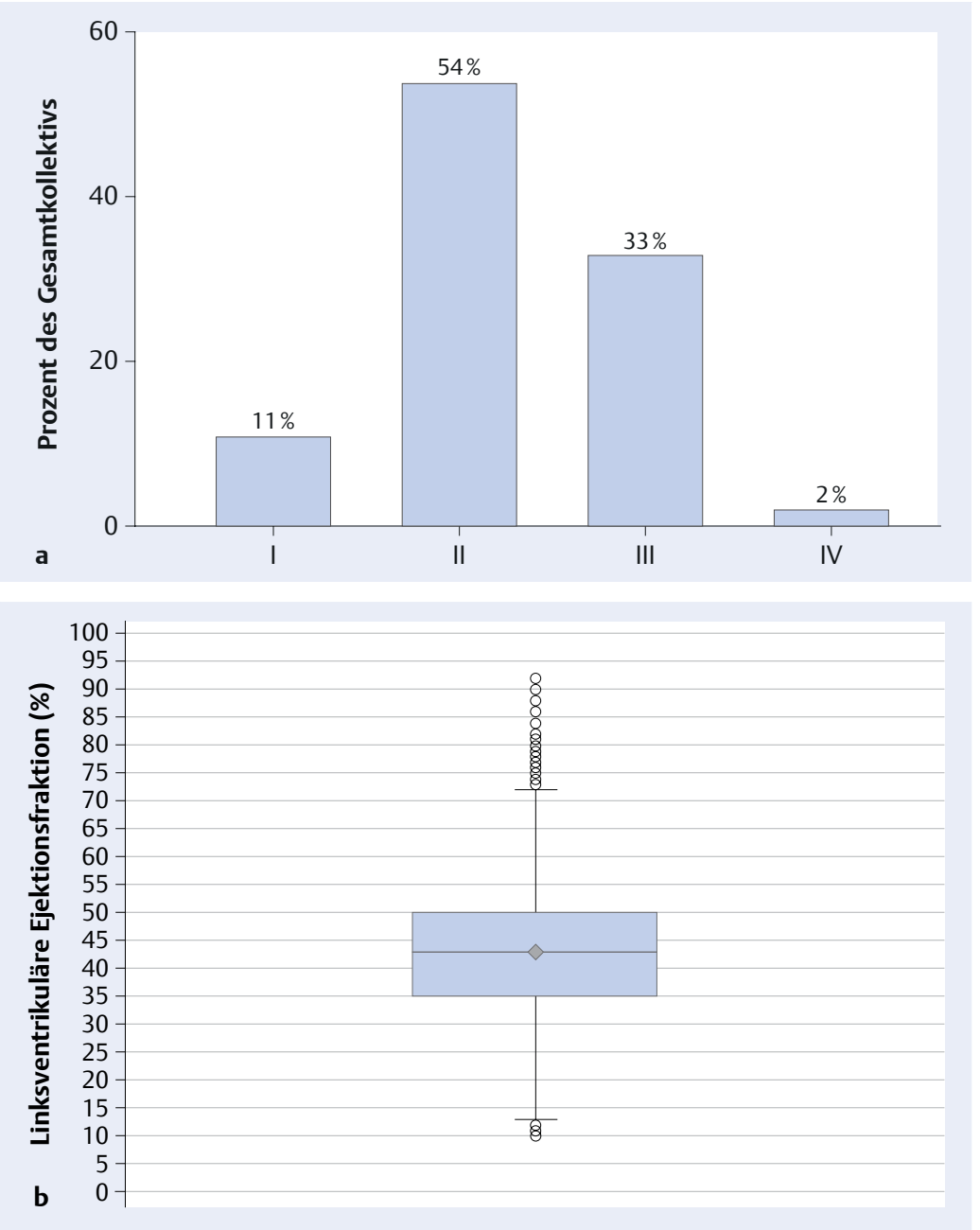

chende Umsetzung der Leitlinienempfehlungen zur pharmakologischen Behandlung von Patienten mit chronischer Herzinsuffizienz in der Praxis, unter anderem auch eine ungenügende Kontrolle der Herzfrequenz.

Das IMPACT-Reco-Programm hat in zwei Studien die Realität der Verschreibung von Pharmaka bei Patienten mit stabiler systolischer Herzinsuffizienz in Frankreich untersucht. Von September 2004 bis März 2005 wurden 1917 und von September 2005 bis März 20061974 ambulante Patienten analysiert [2]. Der Anteil mit Betablocker behandelter Patienten betrug in der ersten Erhebung $65 \%$, in der zweiten Analyse dann bereits $70 \%$. Auch die mittlere Dosierung der Betablocker nahm in diesem Zeitraum zu.

Die wichtigen Ergebnisse der IMPROVE-HF Studie aus dem Jahre 2011 zeigen eine Steigerung des ambulanten Patientenanteils unter Betablocker-Therapie von 85,8\% auf 92,6\% innerhalb des Studienzeitraums von 24 Monaten und weisen darauf hin, dass sich die Leitlinienadhärenz in der Therapie der Herzinsuffizienz in der Praxis steigern lässt durch

- Einsatz spezifischer Entscheidungshilfen,

- Weiterbildung der Ärzte,

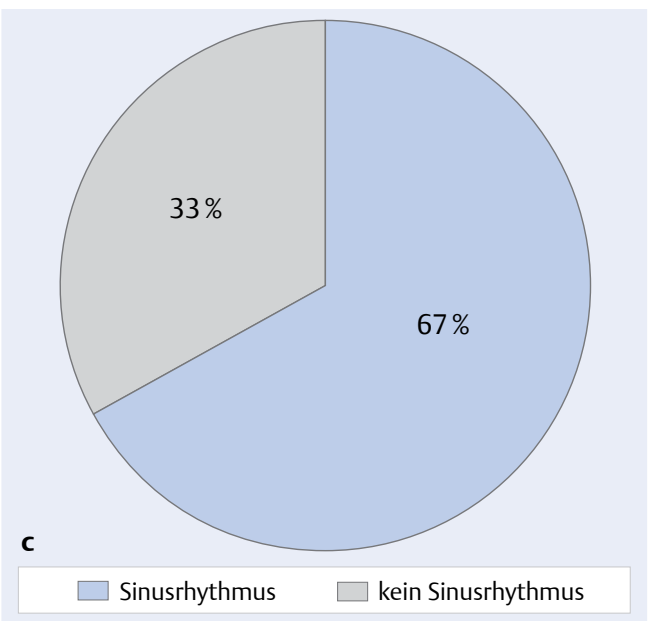

Abb. 1 (a) Verteilung der NYHA-Klassen (als Prozent des Gesamtkollektivs). (b) Boxplot der LVEF (\%) im INDICATEKollektiv (Box: Interquartilabstand 25\%-75\%; horizontale Linie: Median; Raute: arithmetischer Mittelwert; vertikale Linien: Werte innerhalb des 1.5-fachen Interquartilabstands; Kreise: Werte außerhalb des 1,5-fachen Interquartilabstands). (c) Sinusrhythmus im INDICATE-Kollektiv (als Prozent des Gesamtkollektivs).

- strukturierte Erhebung von Patientendaten und - Performance-Feedback [11].

Der ausgeprägte positive Effekt einer strengen Guideline-Adhärenz der Pharmakotherapie hinsichtlich einer Mortalitätsreduktion konnte in weiteren Studien sowohl im klinischen [19] als auch im ambulanten [14] Setting gezeigt werden. Allerdings wurde beispielsweise weder in IMPACT-Reco noch in IMPROVE-HF eine Reduktion der Herzfrequenz erreicht. Trotz Steigerung des Anteils von Patienten unter Betablocker-Therapie, sistierte die mittlere Ruheherzfrequenz in diesen Kollektiven bei Werten $>70 \mathrm{~min}^{-1}$.

Um das therapeutische und prognostische Potenzial, welches in Deutschland in einer optimierten Kontrolle der Herzfrequenz entsprechend den aktuellen Leitlinien besteht, genauer zu erfassen, wurde die multizentrische, bundesweite Querschnitterhebung INDICATE (ScreenINg of toDay's patlents with Chronic systolic heArT failurE) durchgeführt.

\section{Methode}

An der Erhebung nahmen 793 kardiologische Facharztpraxen teil. Zwischen Februar und Juni 

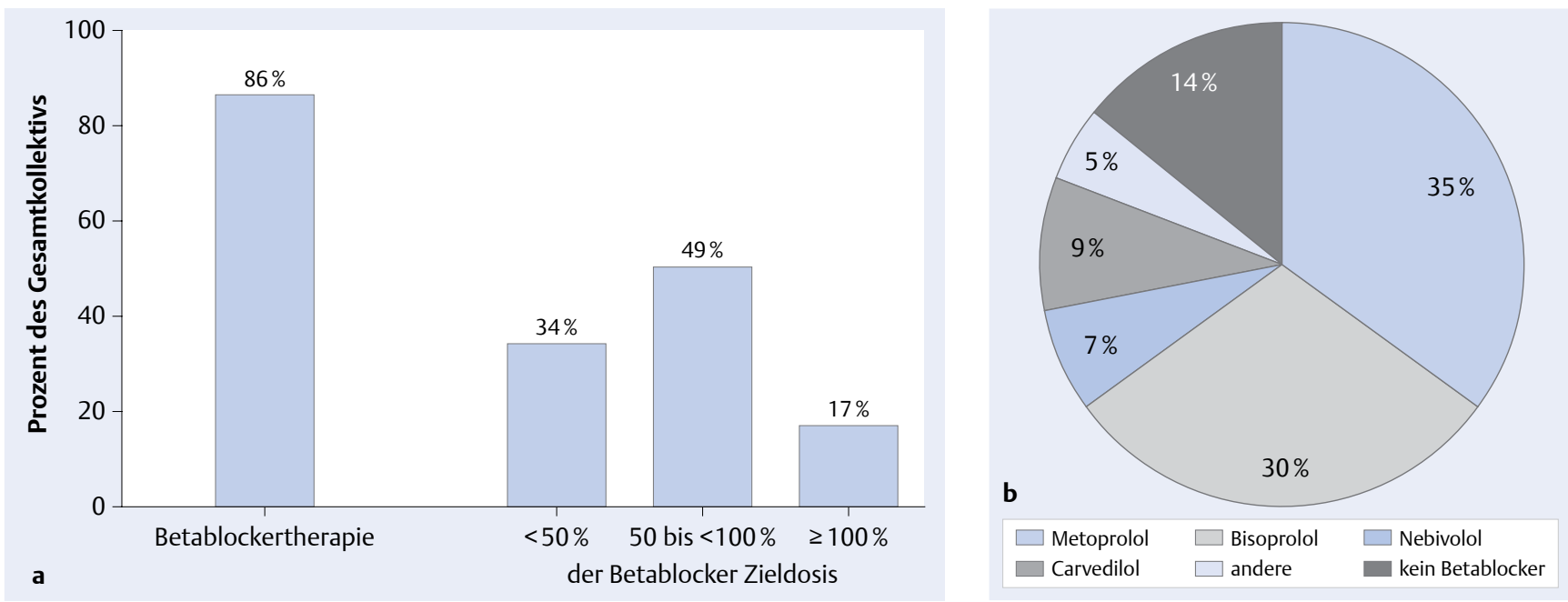

Abb. 2 (a) Anteil von Patienten mit Betablocker-Therapie im Gesamtkollektiv (links) bzw. aufgeschlüsselt nach erreichter Dosis (rechts). (b) Prozentualer Einsatz verschiedener Betarezeptorblocker in der Therapie. Werte angegeben als Prozent des Gesamtkollektivs bzw. Prozent der Patienten mit Betablockertherapie.

2012 sollte jede Praxis 20 konsekutive Patienten mit chronisch systolischer Herzinsuffizienz einschließen. Als einziges prädefiniertes Einschlusskriterium wurde festgelegt, dass pro Studienzentrum 20 konsekutive Patienten mit (echokardiografisch) diagnostizierter und dokumentierter linksventrikulärer Dysfunktion in die Studie aufgenommen werden. Das Protokoll erforderte nicht zwingend eine aktuell durchgeführte echokardiografische Messung der linksventrikulären Ejektionsfraktion, sondern ließ auch vorhergehende echokardiografische Befunde für die Auswertung zu, sofern diese nicht älter als höchstens sechs Monate waren.

Die Messung der Ruheherzfrequenz erfolgte mittels Elektrokardiogramm (EKG) oder durch manuelle, palpatorische Pulsmessung. Dabei wurden nach je fünfminütigen Ruhephasen zwei Pulsmessungen über 30 Sekunden durchgeführt, aus denen anschließend der Mittelwert errechnet wurde. Da die Versorgungsrealität adäquat widergespiegelt werden sollte, wurden keine speziellen Ausschlusskriterien definiert.

Die Dokumentation im Rahmen dieser Querschnitterhebung erfasste:

- demographische Daten

- anamnestische Angaben zur chronischen Herzinsuffizienz

- wichtige Begleiterkrankungen

- die kardiovaskuläre Begleittherapie

- die individuellen Therapieziele für den Patienten

Die Herzinsuffizienz-spezifischen Informationen aus Anamnese und körperlicher Untersuchung umfassten u.a.:

- die Dauer der Erkrankung

- die Ätiologie

- den Schweregrad der Herzinsuffizienz gemäß Stadium der New York Heart Association (NYHA)
- die linksventrikuläre Ejektionsfraktion (LVEF)

- den Blutdruck

- die Herzfrequenz

- Zeichen einer Dekompensation und die Konzentration von brain natriuretic peptide (BNP) im Plasma

Betablockerdosierungen wurden in Äquivalenzdosen umgerechnet. Die von den Leitlinien empfohlene maximale Zieldosis betrug dementsprechend $100 \%$ der Äquivalenzdosis [9]. Um den Effekt der therapeutisch eingesetzten Betablockerdosierungen genauer zu untersuchen, wurde nach drei Dosisbereichen ausgewertet: 0 bis $<50 \%, 50$ bis $<100 \%$, und $\geq 100 \%$ der Zieldosis. Die Daten sind als Mittelwert $( \pm$ Standardabweichung) und $\mathrm{n}$ (Prozent) berichtet. Darüber hinaus werden auch gängige Boxplot-Grafiken mit Darstellung von Mittelwert, Median, Quartilen, Extremwerten verwendet.

Es wurde eine rein deskriptive Auswertung der erhobenen Daten ohne statistische Testungen durchgeführt, da keine prädefinierten Hypothesen überprüft werden sollten.

\section{Ergebnisse}

Insgesamt wurden im Erhebungszeitraum 15148 Patienten im Alter von durchschnittlich 70,2 \pm 11 Jahren (Minimum: 17 Jahre; Maximum: 99 Jahre) analysiert. Davon waren 62\% männlichen Geschlechts. Die Diagnose der chronischen Herzinsuffizienz war bei $88 \%$ der Patienten seit mehr als sechs Monaten bekannt. Eine ischämische Ätiologie lag bei $63 \%$ und eine nicht-ischämische Ätiologie bei 35\% der Patienten vor. Bei 2\% der Patienten trafen Anteile beider Ätiologien zusammen.

Die aktuelle kardiovaskuläre Medikation der Patienten zum Erhebungszeitpunkt wurde de- 
tailliert erfasst, wobei der größte Teil der Patienten mit

- ACE-Hemmern oder AT1-Rezeptorantagonisten $(88 \%)$,

- Betablockern (86\%) und

- Diuretika (77\%) therapiert war ( Tab. 1). Die überwiegende Mehrheit des Gesamtkollektivs wies eine Herzinsuffizienz im NYHA-Stadium II (54\%) oder III (33\%) auf. 27\% der Patienten zeigten eine linksventrikuläre Ejektionsfraktion (LVEF) $\leq 35 \% .67 \%$ des Kollektivs befanden sich im Sinusrhythmus ( Abb. 1), während in der Subgruppe mit Herzfrequenz $\geq 75 \mathrm{~min}^{-1}$ dieser Anteil niedriger lag als in der Subgruppe mit Herzfrequenz $<75 \mathrm{~min}^{-1}$ (63\% vs. 70\%). Eine Betablockertherapie erhielten $86 \%$ der Patienten. If-Kanalblocker (Ivabradin) waren zum Zeitpunkt der Datenerhebung in der Therapie der Herzinsuffizienz noch nicht etabliert und verwendet.

Die aktuellen Leitlinien empfehlen, die individuelle Herzinsuffizienztherapie nicht nur hinsichtlich der Substanzklassen, sondern insbesondere auch im Hinblick auf die anzustrebenden Zieldosierungen zu optimieren. Die BetablockerDosierung betrug bei $49 \%$ der Patienten 50\% bis $<100 \%$ der empfohlenen Zieldosis; bei $34 \%$ lag sie unter $50 \%$; $17 \%$ des Kollektivs wurden mit $100 \%$ oder mehr der Zieldosis behandelt. Am häufigsten wurde Metoprolol eingesetzt, gefolgt von Bisoprolol, Carvedilol, Nebivolol und anderen Substanzen ( $\bullet$ Abb. 2 ).

Die Ruheherzfrequenz im Gesamtkollektiv betrug im Mittel $73,4 \pm 13 \mathrm{~min}^{-1}$. Bei Patienten, die mit weniger als $50 \%$ der Zieldosis behandelt wurden, lag die durchschnittliche Herzfrequenz bei $72,1 \pm 13 \mathrm{~min}^{-1}$, bei Patienten, die $50 \%$ bis $<100 \%$ der Zieldosis erhielten, lag sie bei 73,2 $\pm 13 \mathrm{~min}^{-1}$ und bei Patienten, die mindestens die Zieldosis erhielten, bei 74,2 $\pm 13 \mathrm{~min}^{-1}$ ( $\bullet$ Abb. 3). Eine Herzfrequenz von $\geq 75 \mathrm{~min}^{-1}$ wiesen $42 \%$ des Gesamtkollektivs auf. In dieser Subgruppe erhielten

- $18 \%$ mindestens $100 \%$ der Zieldosis,

- $50 \%$ zwischen $50 \%$ und $<100 \%$,

- und $32 \%$ weniger als $50 \%$ der Zieldosis ( Abb. 4 ). Bezogen auf das Gesamtkollektiv von INDICATE wiesen $27 \%$ der Patienten eine Herzfrequenz $\geq 75 \mathrm{~min}^{-1}$ auf, obwohl sie mindestens $50 \%$ der Betablocker-Zieldosis erhielten, während $38 \%$ aller Patienten unter dieser Dosierung eine Herzfrequenz $<75 \mathrm{~min}^{-1}$ zeigten.

\section{Diskussion}

Mit der Aktualisierung der europäischen Leitlinie zur Diagnose und Therapie der Herzinsuffizienz [9] im Jahr 2012 hat die Ruheherzfrequenz als zu kontrollierender Risikofaktor bei Herz-

Abb. 3 Boxplots der Ruheherzfrequenzen (als Schläge pro Minute) in (a) der Gesamtkohorte, (b-d) in den Subgruppen der jeweils erreichten Dosis der Betarezeptorenblocker im Verhältnis zur empfohlenen Zieldosierung. (Box: Interquartilabstand 25\%-75\%; horizontale Linie: Median; Raute: arithmetischer Mittelwert; vertikale Linien: Werte innerhalb des 1.5-fachen Interquartilabstands; Kreise: Werte außerhalb des 1,5-fachen Interquartilabstands.)
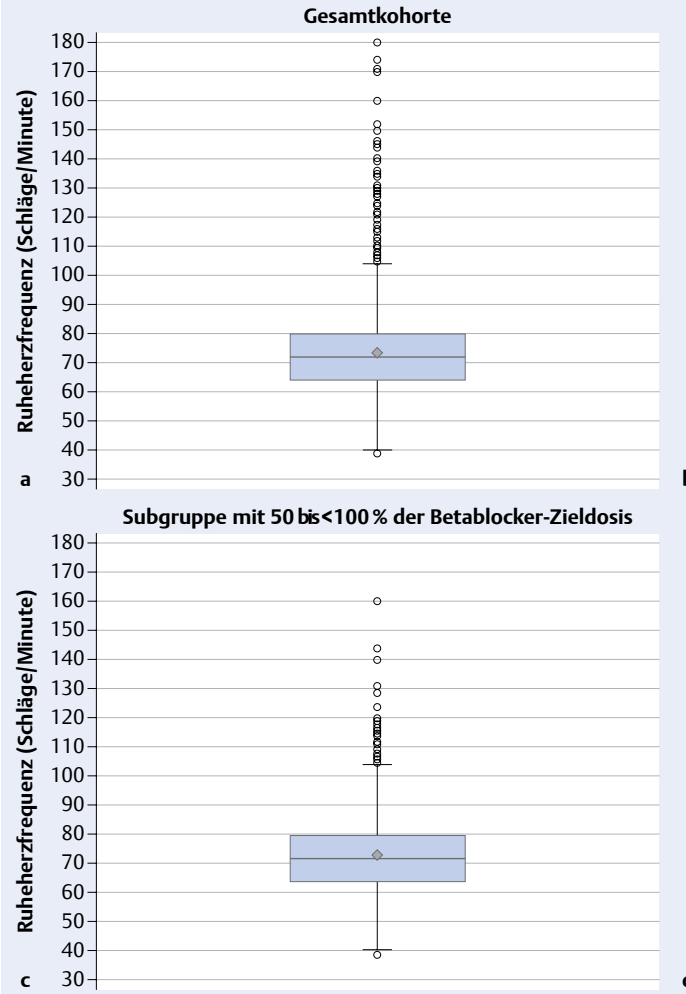

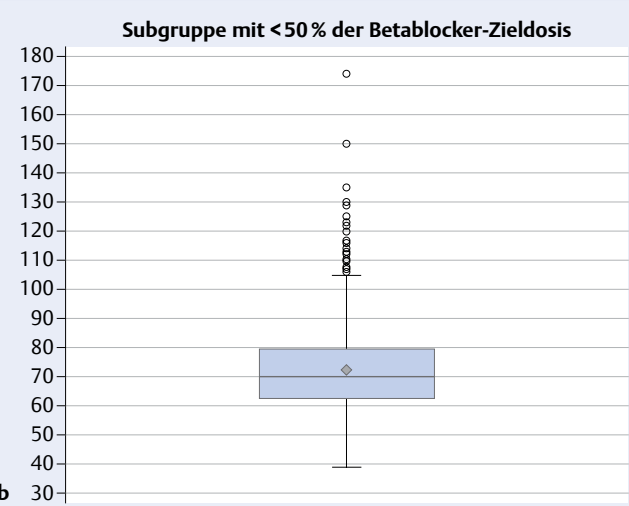

Subgruppe mit $\geq 100 \%$ der Betablocker-Zieldosis

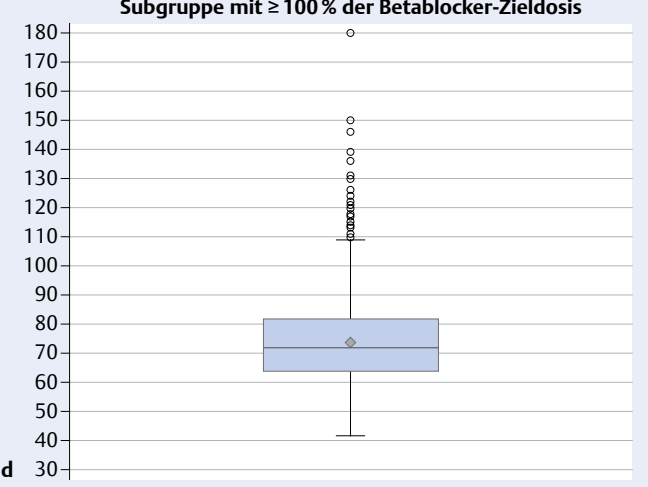


insuffizienz-Patienten deutlich an Bedeutung gewonnen. Ziel der vorliegenden Querschnitterhebung war es, die Pharmakotherapie in einem Kollektiv von mehr als 15000 kardiologisch betreuten Patienten im Hinblick auf eine adäquate Kontrolle der Ruheherzfrequenz zu evaluieren. Im Fokus der Betrachtung standen dabei das Ausmaß und die Dosierung der Betablocker-Therapie.

INDICATE bestätigt, dass in Deutschland der Einsatz von Betablockern bei Patienten mit chronischer systolischer Herzinsuffizienz in der Praxis mittlerweile sehr gut etabliert ist: $86 \%$ des Kollektivs wurden entsprechend behandelt. Ähnliche Anteile wurden in jüngeren Registerstudien ermittelt. In der ESC-HF Pilot-Studie [8] erhielten etwa $90 \%$ der Patienten Betarezeptorenblocker, in der zweiten Erhebung der IMPROVE-HF-Studie [11] $93 \%$. Einen relativ niedrigen Anteil von 70\% fand dagegen das französische IMPACT-Reco-Programm, das aber bereits in den Jahren 2005 und 2006 durchgeführt wurde [2].

Trotz des hohen Anteils von Patienten mit Betablocker-Therapie war die Herzfrequenz bei $42 \%$ der Patienten in INDICATE nicht optimal kontrolliert $\left(\geq 75 \mathrm{~min}^{-1}\right)$. Dieser Zusammenhang war unabhängig von der eingenommenen Betablockerdosis. Vielmehr zeigte sich sogar die Tendenz, dass mit mindestens Betablocker-Zieldosis $(\geq 100 \%)$ behandelte Patienten eine höhere Herzfrequenz aufwiesen als diejenigen der mittleren $(50 \%$ bis $<100 \%)$ und niedrigen Kategorie $(<50 \%)$. In allen drei untersuchten Dosisbereichen lag die mittlere Ruheherzfrequenz über dem von aktuellen europäischen Leitlinien empfohlenen Schwellenwert von $70 \mathrm{~min}^{-1}[9]$.

In INDICATE waren $88 \%$ der Patienten seit mindestens sechs Monaten an Herzinsuffizienz erkrankt. Dies bedeutet, dass bei vielen Patienten die Auftitrationsphase der Betablocker-Medikation wohl bereits abgeschlossen und keine weitere Aufdosierung geplant war. Da die Erhebung nicht als Titrationsstudie konzipiert war, wurden mögliche Gründe für das Nicht-Erreichen der Betablocker-Zieldosis nicht explizit erfasst.

Die longitudinalen Registerstudien IMPROVE-HF [11] und IMPACT-Reco [2] geben zudem Hinweise, dass eine Zunahme des Anteils von Patienten, die Betablocker erhalten, nicht unbedingt einen Einfluss auf die adäquate Kontrolle der Herzfrequenz hat. So war in IMPROVE-HF der Anteil von Patienten mit Betablocker-Therapie von $86 \%$ auf $93 \%$ gestiegen, die mittlere Herzfrequenz aber mit $72 \pm 11$ versus $72 \pm 12 \mathrm{~min}^{-1}$ unverändert geblieben. Im IMPACT-Reco-Programm hatte sich der Anteil von Patienten mit Betablocker-Therapie von $65 \%$ auf $70 \%$ erhöht, die durchschnittliche Herzfrequenz aber sistierte ebenfalls bei Werten $>70 \mathrm{~min}^{-1}\left(73 \pm 14\right.$ versus $\left.73 \pm 15 \mathrm{~min}^{-1}\right)$.
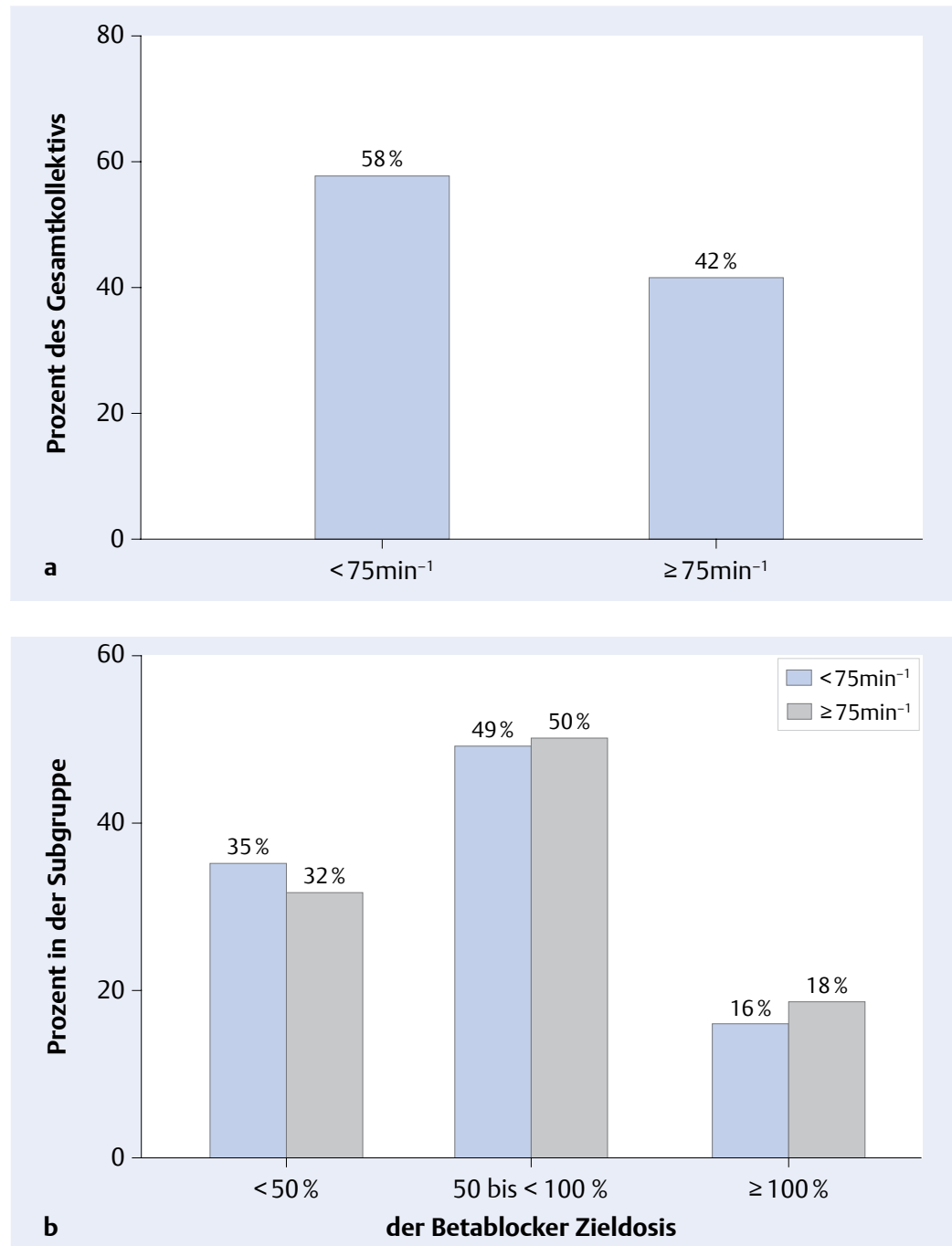
der Betablocker Zieldosis

Eine prospektive Kohortenstudie von Franke et al. bestätigt, dass die Ruheherzfrequenz von Patienten mit Herzinsuffizienz trotz optimierter Betablockertherapie häufig nicht ausreichend gesenkt werden kann. Die Autoren schlossen in ihre Analyse 443 Patienten mit einer linksventrikulären Ejektionsfraktion $\leq 35 \%$, Sinusrhythmus und einer Herzinsuffizienz im NYHA-Stadium II-IV ein [6]. Beim in dieser Studie forciert angestrebten Auftitrieren erreichten $69 \%$ der Patienten mindestens die Hälfte der Betablocker-Zieldosis und 29\% die volle Dosis. Dennoch wiesen 53\% der Patienten am Ende der Auftitration eine Herzfrequenz von $\geq 75 \mathrm{~min}^{-1}$ auf.

Ähnliche Ergebnisse im Hinblick auf das Erreichen der Zieldosierungen zeigt auch die kürzlich publizierte prospektive Beobachtungsstudie der ESC Heart Failure Long-Term Registry mit Daten aus 21 europäischen Ländern [7]. Dort wurden 89\% der eingeschlossenen Patienten mit chronischer Herzinsuffizienz $(n=7.401)$ mit Betablockern behandelt, wobei $56 \%$ der Patienten eine Herzfrequenz $\geq 70 \mathrm{~min}^{-1}$ aufwiesen. Insgesamt erreichten in dieser großen Untersuchung 18\% des Kollektivs die empfohlene Zieldosis. Dieser Wert deckt sich
Abb. 4 (a) Anteil von Patienten im Gesamtkollektiv mit Herzfrequenzen $<75 \mathrm{~min}^{-1}$ bzw. $\geq 75 \mathrm{~min}^{-1}$. (b) Aufgeschlüsselt nach der erreichten Betablocker-Dosis in der jeweiligen Frequenz-Subgruppe. Werte angegeben als Prozent des Gesamtkollektivs bzw. Prozent der Patienten in der jeweiligen Frequenz-Subgruppe. 
gut mit den Ergebnissen aus INDICATE (17\% mit mindestens Zieldosis). Die Verantwortlichen der ESC Heart Failure Long-Term Registry fordern deshalb auch eine bessere Berücksichtigung der realen Möglichkeiten bezüglich der Erreichbarkeit von in klinischen Guidelines formulierten Vorgaben und Empfehlungen. Dieses in Beobachtungsstudien dokumentierte „real-life“-Szenario sollte bei der Erstellung von Guidelines, die primär auf Ergebnissen kontrollierter klinischer Studien beruhen, laut den Autoren in jedem Fall in entsprechender Form mit einfließen.

Zusammenfassend zeigt die vorliegende Erhebung, dass sich in der klinischen Praxis eine Senkung der Ruheherzfrequenz in den empfohlenen Bereich trotz der mittlerweile sehr guten Akzeptanz der Betablocker in der Herzinsuffizienztherapie schwierig gestalten kann, unter anderem durch pulmonale Begleiterkrankungen und hypotensive Phasen in einem häufig multimorbiden, betagten Patientenkollektiv.

Sehr aktuelle Registerdaten von Maggioni et al [7] geben einen guten Überblick über die Gründe für die Nicht-Anwendung von evidenzbasierten Therapien, u.a. Betablockern. Bei den Kontraindikationen wurden dabei am häufigsten genannt:

- Asthma/COPD

- Bradyarrhythmien

- Hypotension

Die gängigsten Intoleranzen ließen sich zurückführen auf:

- Bronchospasmen

- symptomatische Hypotonie

- Bradykardien

Eine Auftitration der Betablockerdosis auf empfohlene Zieldosen ist deshalb ebenfalls häufig nicht möglich oder sogar kritisch.

Aus INDICATE wird darüber hinaus deutlich, dass selbst bei Erreichen von hohen Betablockerdosierungen eine ausreichende Senkung der Ruheherzfrequenz in prognostisch günstige Bereiche nicht in jedem Fall gewährleistet sein kann. Dass die durch eine adäquate Betablockertherapie erreichbare Frequenzreduktion nicht ausschließlich auf linearen Dosiseffekten beruht, wurde in einer post-hoc Subgruppenanalyse aus MERIT-HF gezeigt, wo etwa $30 \%$ der Patienten bereits unter niedrigeren Dosen von Metoprolol ( $\leq 100 \mathrm{mg} / \mathrm{Tag})$ maximale Herzfrequenzreduktionen in vergleichbarer Größenordnung und ähnliche prognostische Verbesserungen im Hinblick auf Reduktion der Gesamtmortalität wie die MetoprololHochdosisgruppe (>100 mg/ Tag) aufwiesen [18].

Bei ungenügender Reduktion der Herzfrequenz sollte unabhängig von der bereits zugrundeliegenden Betablockerdosierung - im Rahmen der zugelassenen Indikation (Sinusrhythmus, NYHAStadium II-IV, Ruheherzfrequenz $\geq 75 \mathrm{~min}^{-1}$ ) - die zusätzliche Frequenzsenkung mit Ivabradin in
Betracht gezogen werden [16]. Auch unter Berücksichtigung eines bestehenden Sinusrhythmus bei $67 \%$ der dokumentierten ambulanten Patienten als Voraussetzung für einen Einsatz der Substanz (27\% des Gesamtkollektivs mit diagnostiziertem Vorhofflimmern) ergibt sich hier ein großes therapeutisches Potential.

Die Therapieerweiterung mit Ivabradin hat nachgewiesenermaßen günstige prognostische Effekte, zusätzlich zur bestehenden Vortherapie mit ACE-Hemmer / AT1-Rezeptorantagonisten, Betablockern und Aldosteronantagonisten [1, 15], und wird durch die aktuellen europäischen Herzinsuffizienz-Leitlinien gestützt [9]. Es ist anzumerken, dass sehr aktuell publizierte Daten zu der großen randomisierten, placebo-kontrollierten SIGNIFYStudie bei Patienten mit koronarer Herzkrankheit (KHK) und erhaltener linksventrikulärer Funktion (d.h. ohne klinische Herzinsuffizienz) keinen signifikanten prognostischen Nutzen einer Herzfrequenzreduktion mit Ivabradin zeigen konnten [5]. Im Rahmen von INDICATE lag der Fokus allerdings in Anlehnung an die SHIFT-Studie auf der Erfassung von Patienten mit chronischer Herzinsuffizienz, nicht auf einem reinen KHK-Kollektiv.

Bei der Interpretation der INDICATE-Daten sind einige dem Studiendesign geschuldete Limitationen zu beachten. Das Protokoll erforderte nicht zwingend eine aktuell durchgeführte echokardiographische Messung der linksventrikulären Ejektionsfraktion, sondern ließ auch historische echokardiographische Befunde für die Auswertung zu. Die punktuelle Querschnitterhebung spiegelt nur den aktuellen Ist-Zustand wider und erlaubt somit keinen Vergleich über die Zeit hinweg. Die Messung der Ruheherzfrequenz erfolgte nicht ausschließlich - wie beispielsweise im Rahmen der SHIFT-Studie - mittels EKG, sondern häufig auch durch manuelle Pulsmessung (19\% der Patienten). Andererseits besitzt aber gerade das vorliegende Studiendesign auch Stärken: das pragmatische Design erlaubte den raschen Einschluss einer sehr großen Anzahl von Patienten, so dass ein Patientenquerschnitt erreicht wurde, der das Klientel von Patienten mit chronischer systolischer Herzinsuffizienz in der klinischen Praxis repräsentativ abbildet.

\section{Schlussfolgerung}

INDICATE, eine bundesweite Querschnitterhebung bei ambulanten Patienten mit chronisch systolischer Herzinsuffizienz zeigt, dass ein erheblicher Anteil dieser Patienten trotz Betablocker-Therapie - unabhängig von der Dosierung - eine erhöhte Ruheherzfrequenz $\left(\geq 75 \mathrm{~min}^{-1}\right)$ aufweist. INDICATE unterstreicht somit das Potenzial einer Leitlinien-orientierten pharmakologischen Optimierung der Ruheherzfrequenz bei Patienten mit chronisch systolischer Herzinsuffi- 
zienz. Dies ist insbesondere bedeutsam im Hinblick auf die Reduktion von (Re-)Hospitalisierungen und kardiovaskulärer Mortalität bei diesem Patientenkollektiv.

\section{Konsequenz für Klinik und Praxis}

- Eine erhöhte Ruheherzfrequenz ist ein wichtiger Faktor, der die Morbidität und Mortalität von Patienten mit chronischer Herzinsuffizienz steigert und therapeutisch gut beeinflussbar ist.

- Der größte Teil der ambulanten Patienten mit chronisch systolischer Herzinsuffizienz wird in Deutschland mit Betablockern therapiert.

- Trotz bestehender Betablocker-Therapie weisen $42 \%$ der ambulant behandelten Patienten eine erhöhte Ruheherzfrequenz $\left(\geq 75 \mathrm{~min}^{-1}\right)$ auf.

- Patienten mit höheren Betablocker-Dosierungen weisen in INDICATE eine tendenziell höhere Herzfrequenz auf als Patienten mit niedrigeren Dosierungen.

- Bei nicht ausreichender Frequenzsenkung durch eine alleinige Gabe von Betablockern stehen weitere leitlinienkonforme Therapieoptionen zur Verfügung, die genutzt werden sollten.

\section{Danksagung}

Die Autoren danken ausdrücklich allen bundesweit teilnehmenden Ärzten für ihren substanziellen Beitrag bei der Erhebung der vorgestellten Daten. Die INDICATE-Studie wurde finanziell unterstützt von Servier Deutschland $\mathrm{GmbH}$, München.

\section{Literatur}

1 Böhm M et al. Heart rate at baseline influences the effect of ivabradine on cardiovascular outcomes in chronic heart failure: analysis from the SHIFT study. Clin Res Cardiol 2012; 102: 11-22

2 De Groote P, Isnard R, Clerson P et al. Improvement in the management of chronic heart failure since the publication of the updated guidelines of the European Society of Cardiology - The Impact-Reco Programme. Eur J Heart Fail 2009; 11: 85-91

3 Fischer M, Baessler A, Holmer SR et al. Epidemiologie der linksventrikulären systolischen Dysfunktion in der Allgemeinbevölkerung Deutschlands. Z Kardiol 2003; 92: 294-302

4 Foody JM, Farrell MH, Krumholz HM. Beta-Blocker therapy in heart failure; scientific review. JAMA 2002; 287: 883-889

5 Fox K, Ford I, Steg PG et al. Ivabradine in stable coronary artery disease without clinical heart failure. N Engl J Med 2014; 371:1091-1099
6 Franke J, Wolter JS, Meme L et al. Optimization of pharmacotherapy in chronic heart failure: is heart rate adequately addressed? Clin Res Cardiol 2013; 102: 23-31

7 Maggioni AP, Anker SD, Dahlström U et al. Are hospitalized or ambulatory patients with heart failure treated in accordance with European Society of Cardiology guidelines? Evidence from 12440 patients of the ESC Heart Failure Long-Term Registry. Eur J Heart Fail 2013; 15: 1173-1184

8 Maggioni AP, Dahlström U, Filippatos G et al. EURObervational Research Programme: regional differences and 1-year follow-up results of the Heart Failure Pilot Survey (ESC-HF Pilot). Eur J Heart Fail 2013; 15: 808-817

9 McMurray J et al. ESC Guidelines for the diagnosis and treatment of acute and chronic heart failure 2012 Eur Heart ] 2012; 33: 1787-1847

10 Neumann T, Biermann J, Neumann A et al. Heart failure: the commonest reason for hospital admission in Germany. Dtsch Arztebl Int 2009; 106: 269-275

11 O'Connor CM, Albert NM, Curtis AB et al. Patient and Practice Factors Associated with improvement in use of guideline-recommended therapies for outpatients with heart failure (from the IMPROVE HF trial). Am J Cardiol 2011; 107: 250-258

12 Pocock S], Wang D, Pfeffer MA et al. Predictors of mortality and morbidity in patients with chronic heart failure. Eur Heart ] 2006; 27: 65-75

13 Reil JC Custodis F, Swedberg K et al. Heart rate reduction in cardiovascular disease and therapy. Clin Res Cardiol 2011; 100: 11-19

14 Störk S, Hense HW, Zentgraf C et al. Pharmacotherapy according to treatment guidelines is associated with lower mortality in a community-based sample of patients with chronic heart failure. A prospective cohort study. Eur J Heart Fail 2008; 10: 1236-1245

15 Swedberg K, Komajda M, Böhm M et al. Ivabradine and outcomes in chronic heart failure (SHIFT): a randomised placebo-controlled study. Lancet 2010; 376: 875-885

16 Swedberg K, Komajda M, Böhm M et al. Effects on outcomes of heart rate reduction by ivabradine in patients with congestive heart failure: is there an influence of beta-blocker dose? Findings from the SHIFT (Systolic Heart failure treatment with the I(f) inhibitor ivabradine Trial) study. J Am Coll Cardiol 2012; 59 : 1938-1945

17 Tavazzi L, Senni M, Metra M et al. Multicenter prospective observational study on acute and chronic heart failure: one-year follow-up results of IN-HF (Italian Network on Heart Failure) outcome registry. Circ Heart Fail 2013; 6: 473-481

18 Wikstrand J, Hjalmarson A, Waagstein F et al. Dose of Metoprolol CR/XL and clinical outcomes in patients with heart failure. J Am Coll Cardiol 2002; 40: 491-498

19 Zugck C, Franke J, Gelbrich G et al. Implementation of pharmacotherapy guidelines in heart failure: experience from the German Competence Network Heart Failure. Clin Res Cardiol 2012; 101: 263-272
Interessenkonflikt

C.Z., C. T. und S.S. geben an, Honorare für Beratertätigkeiten und Vorträge und finanzielle Unterstützung für Forschungsprojekte von Servier Deutschland $\mathrm{GmbH}$ (München) erhalten zu haben.

P. M. und G.S. geben an, Mitarbeiter der medizinischwissenschaftlichen Abteilung bei Servier Deutschland GmbH (München) zu sein. 